\title{
A follow-up study of the in-practice results of pulsed electromagnetic field therapy in the management of nonunion fractures
}

This article was published in the following Dove Press journal:

Orthopedic Research and Reviews

I December 2016

Number of times this article has been viewed

\author{
Hallie B Murray' \\ Brian A Pethica ${ }^{1,2}$ \\ 'EBI, LLC (a Zimmer Biomet \\ company), Parsippany, NJ, USA; \\ ${ }^{2}$ Department of Chemical and \\ Biological Engineering, Princeton \\ University, Princeton, NJ, USA
}

Correspondence: Hallie B Murray EBI, LLC (a Zimmer Biomet company), 399 Jefferson Road, Parsippany, NJ 07054, USA

Tel + I 9732999300

Fax + I 9738871347

Email hallie.murray@zimmerbiomet.com
Abstract: During normal fracture repair, healing occurs within a few months. However, for a minority of patients, the processes of bone repair are compromised or interrupted leading to the development of delayed union and nonunion fractures. Noninvasive bone growth stimulators using pulsed electromagnetic field (PEMF) technology are currently in widespread use by patients with impaired fracture healing. This article reports the results of a follow-up study of 1,382 patients treated with PEMF stimulation to evaluate success rates and the relationship between average daily use and the clinical outcomes of therapy as reported by their prescribing physicians. The reported overall success rate for the 1,382 patients was $89.6 \%$. The results were analyzed in audited subsets comparing days of treatment time and average daily use of the electrical bone growth stimulator, using several statistical methods. Linear regression analysis indicated a 6-day reduction in time to heal with each additional hour of average daily use. Survival analysis concluded that the median heal time was reduced by $35 \%-60 \%$, depending on the different fracture characteristics of patients who complied with the recommended daily use of 10 hours per day. A third statistical analysis indicated that patients treated with the PEMF device for 9 hours or more per day had a significant reduction in time to heal, achieving successful fracture repair an average of 76 days earlier than patients treated with the PEMF device for an average of 3 hours or less per day. Overall, these different methods of statistical analysis indicate that PEMF therapy correlates with an acceleration in the healing of nonunion fractures. Keywords: PEMF, pulsed electromagnetic field, dose-response, nonunion, time to heal

\section{Introduction}

Musculoskeletal injuries account for $\sim 60 \%$ of the injuries treated in the US. ${ }^{1}$ Of the $\sim 57.2$ million musculoskeletal injuries that occur annually, 15.3 million are fractures. ${ }^{1}$ In the majority of patients who present with a fracture, normal healing occurs within a few months. However, some patients have impaired fracture repair. Delayed union and nonunion are major complications in the treatment of skeletal defects. They affect $400,000-800,000$ patients annually in the US, amounting to an estimated health care cost of \$3-6 billion annually. ${ }^{2}$ Numerous treatment modalities have been designed to combat fracture nonunions including, but not limited to, internal and external fixation devices, bone grafts, bone substitutes, biologics such as platelet extracts and bone morphogenetic proteins (BMPs), and biophysical stimulation including ultrasound and electrical stimulation. Today, $>75,000$ bone growth stimulators are used annually to improve nonunion fracture repair, and this number is expected to continue to rise due to the increasing population of patients aged 65 years and older who are more susceptible to sustaining fracture nonunions. 
Pulsed electromagnetic field (PEMF) stimulation first gained US Food and Drug Administration approval for the treatment of nonunion fractures in $1979 .{ }^{3}$ Since gaining approval, several different studies have evaluated the efficacy of PEMF stimulation in the treatment of fracture nonunions with success rates ranging from $68 \%$ to $90 \%$ depending on the fractures treated. ${ }^{4-14}$ Although these studies found PEMF stimulation to be effective in inducing fracture repair, they did not evaluate the effect of treatment "dose" on patient healing times. Therefore, a study was conducted to collect the clinical data pertaining to the therapeutic use of the EBI ${ }^{\circledR}$ PEMF noninvasive bone growth stimulator to evaluate patient device use and the associated clinical outcomes.

\section{Materials and methods Device description}

The Biomet ${ }^{\oplus}$ EBI Bone Healing System (EBI, LLC (a Zimmer Biomet company), Parsippany, NJ, USA) is a portable, noninvasive PEMF device that induces weak pulsing electrical currents at the fracture site. These currents are generated by a low-energy electromagnetic field consisting of $4.5 \mathrm{~ms}$ bursts of pulses, repeating at $15 \mathrm{~Hz}$, with a peak magnetic field of $18 \mathrm{G}$ during each $225 \mu$ s pulse. The system consists of an electronic control unit with an elapsed real-time clock that measures the total time the device is used and an anatomically configured treatment coil that is applied at the fracture site to deliver the therapeutic treatment.

\section{Data acquisition}

Western Institutional Review Board (WIRB) gave ethical approval for this study. WIRB did not require patient consent be obtained because the study met the conditions for exemption under 45 CFR 46.101(b)(4) (US Department of Health and Human Service Basic Policy for the Protection of Human Research Subjects).

Data were collected from 1,382 consecutive patients throughout the US diagnosed with nonunion fractures (includes all fractures that show no visibly progressive signs of healing [both 9-month and $<9$-month nonunions]) over a 17-month period, who were treated with a PEMF stimulation device as prescribed by their treating physician. Patients were instructed to apply and use their devices for a recommended 10 hours per day. The use of fracture immobilization, nonweight bearing, pain medication, etc was at the discretion of the treating physician. The data from the overall patient data set was first stratified to select patients with fractures of the tibia, tibia/fibula, and scaphoid ( $\mathrm{n}=888$ ) as shown in Figure 1. Patients with fractures of the tibia, tibia/fibula, and scaphoid were chosen because they represented the majority of the fractures treated. Of this subset, 314 had not returned their device and 204 patients were missing either their treatment start date or treatment end date. Therefore, 370 patients had complete data for analysis, comprising treatment outcome, treatment start date, treatment end date, and recorded device use time, giving the time to heal or fail for each patient. The collected patient data were confirmed by the treating

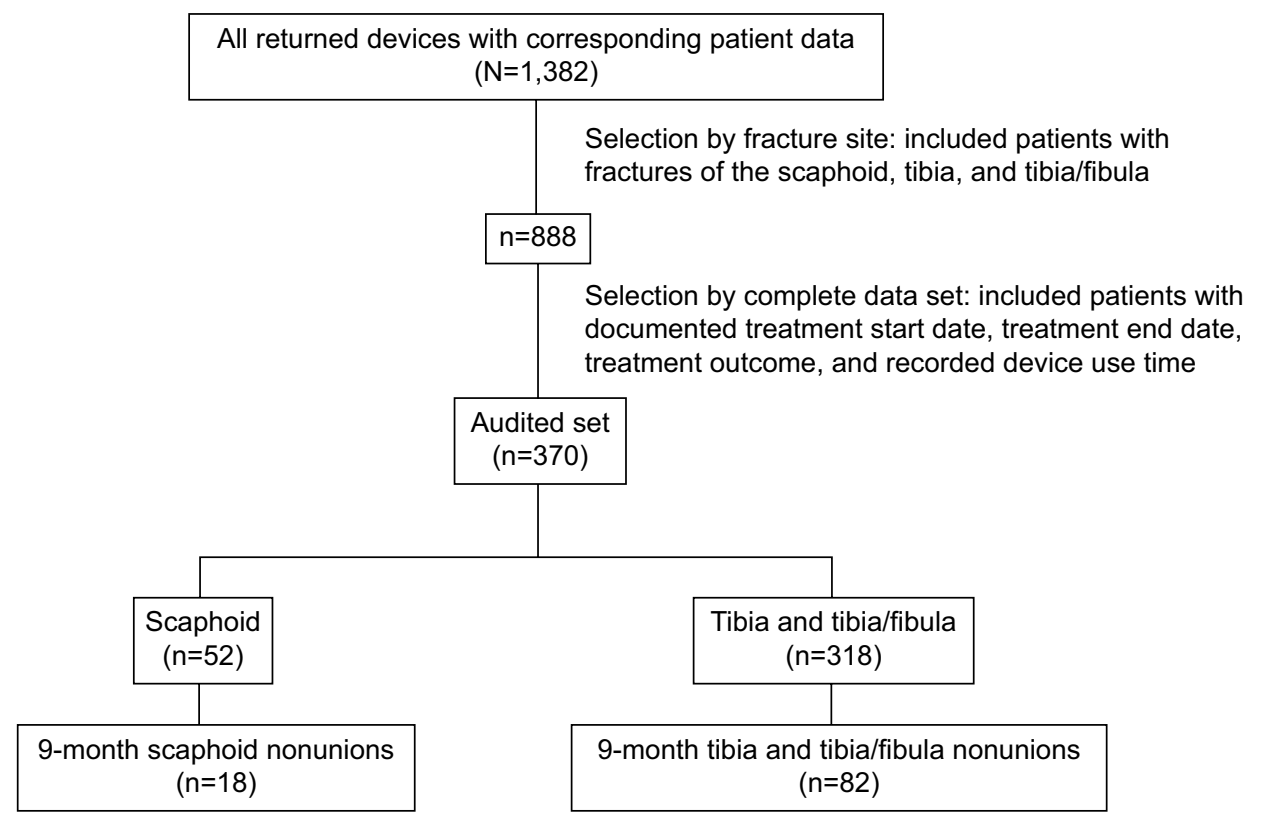

Figure I Data acquisition.

Notes: Schematic of data acquisition from the total patient population $(\mathrm{N}=1,382)$. The data were stratified to show patients with fractures of the tibia, tibia/fibula, and scaphoid $(n=888)$, and from this set a smaller patient subpopulation with complete audited data for analysis was identified $(n=370)$. 
physician through written and telephone correspondence. Additional analysis was performed by further stratifying the audited data set to determine the healing patterns for patients with nonunions persisting for 9 months prior to the initiation of PEMF treatment.

\section{Determination of device use in hours per day}

The number of treatment hours recorded by the device was used in conjunction with the total days of treatment (time from the date of treatment initiation to fracture healing/treatment end date) to determine the average hours of use per day. A dose-response relationship was tested by comparing the device use in hours per day with the total number of days required to obtain successful fracture repair.

\section{Statistical analysis}

Three different statistical analyses were performed to test for a correlation between treatment time and average daily dose, taking the average daily dose as the independent variable. Linear regression and survival analysis methods were applied to cover alternative descriptions of the possible correlations. Multivariate survival analysis was also used to test the statistical effects of other fracture characteristics on fracture healing. In addition, the data were subdivided into groups corresponding to several ranges of average daily use and the differences in time to heal were compared for statistical significance. Results were considered significant at the $P<0.05$ significance level.

\section{Results}

Of the 1,382 patients included in the overall patient data set, 1,238 healed for an overall healing rate of $89.6 \%$ as determined by the treating physician. The audited set of 370 patients with complete data for device usage comprised 252 males and 118 females. Definitive healing outcomes (healed, failed, or went on to surgical intervention) were reported for 330 patients within the audited set. Other reported outcomes included treatment in progress, partial response, lost to follow-up, and patient withdrew. Patients who went on to surgical intervention were considered failures and patients with no definitive healing outcome (ie, partial response, lost to follow-up, and patient withdrew) were not included in the healing analysis. Treatment success as determined by the treating physician was reported for 285 patients for an overall success rate of $86.4 \%$ in the audited patient set.

Table 1 gives a breakdown of patient characteristics in the audited set. Patients were evaluated for their type of fracture, presence of motion, number of prior surgeries,
Table I Patient characteristics for the audited patient set

\begin{tabular}{ll}
\hline Injury type & \\
Closed & $65 \%$ \\
Open & $25 \%$ \\
Other & $2 \%$ \\
Unknown & $8 \%$ \\
Trophic level & \\
Oligotrophic & $69 \%$ \\
Hypertrophic & $13 \%$ \\
Atrophic & $7 \%$ \\
Unknown & $11 \%$ \\
Presence of motion & \\
No & $49 \%$ \\
Yes & $47 \%$ \\
Unknown & $4 \%$ \\
Number of prior surgeries & \\
0 & $68 \%$ \\
I & $20 \%$ \\
2 & $8 \%$ \\
3 & $1 \%$ \\
$4+$ & $3 \%$ \\
Type of fracture immobilization & \\
Plastic cast & $56 \%$ \\
Brace & $10 \%$ \\
Plaster & $9 \%$ \\
External fixation & $1 \%$ \\
None & $22 \%$ \\
Other & $2 \%$ \\
\hline
\end{tabular}

and type of fracture immobilization. To test for potential selection bias between the overall data set and the audited set, a random sample of patients with tibia, tibia/fibula, and scaphoid fractures from the overall data set $(n=888)$ was compared for the same patient factors in the audited set $(n=370)$. Comparison of the two patient populations revealed no significant differences.

Linear regression analysis performed on the healed patients in the audited set revealed that each additional hour of PEMF treatment correlated significantly with a 6-day reduction in the time to heal $(P<0.001)$. No correlation was found for time to reported failure and average daily use when linear regression analysis was performed on the patients who failed to heal $(P<0.605)$. Furthermore, when the linear regression method was applied to the overall set of 1,382 patients with all fractures included, it also showed a statistically significant decrease in average time to heal with increasing average daily use for the 1,238 patients reported as healed $(P<0.001)$. For the 1,238 healed patients, each additional hour of PEMF treatment correlated with a 9-day reduction in time to heal. Similarly, as reported for the audited data set, no such correlation in average daily use was found for the 144 patients reported to have failed healing ( $P=0.622)$.

In a second analysis, subdivision of the data from the audited set into average time-of-use ranges again found that 


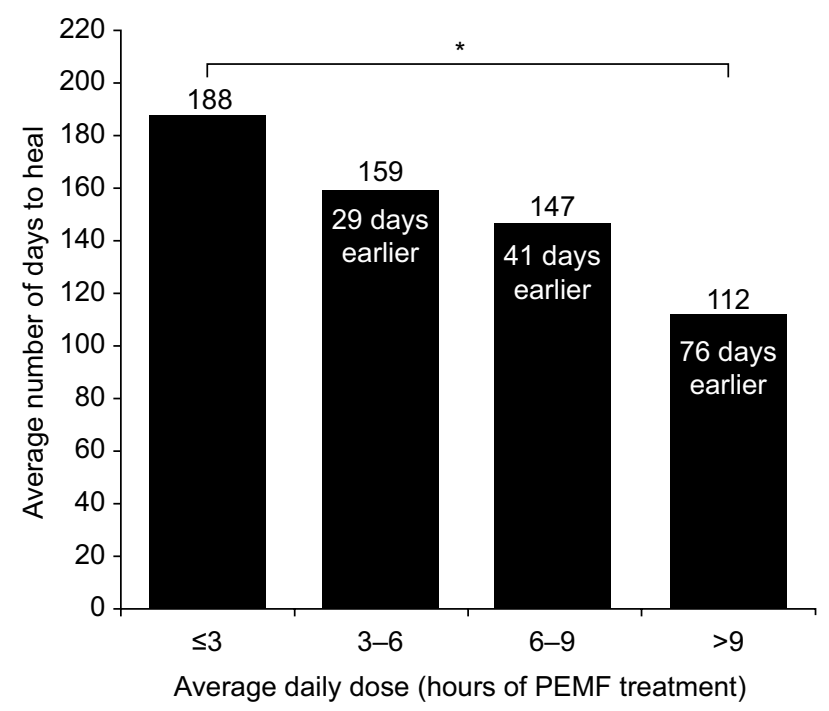

Figure 2 Dose-response relationship for nonunion fractures.

Notes: Average days to heal shown for four groupings of average daily PEMF treatment in patients with nonunion fractures. $*$ indicates a significant difference $(P<0.00 \mathrm{I})$.

Abbreviation: PEMF, pulsed electromagnetic field.

the time to heal decreases as the average daily use increases. Patients who were treated with the device for $>9$ hours per day (representative of compliant use) healed on average 76 days earlier than patients who were treated with their PEMF device for 3 hours or less per day (representative of noncompliant use; $P<0.001$; Figure 2). Moreover, study analysis showed no statistically significant further reduction in the time to heal for the small number of patients who were treated with their PEMF device for $>10$ hours per day, the recommended device treatment use.

Survival analysis was used to estimate the median time to heal as a function of average daily treatment time. Using the survival relation to compare treatment times of 1 hour per day (approximating nonuse) and 10 hours per day (the recommended device use time) shows a reduction in median time to heal by $35 \%-40 \%$ in the scaphoid, tibia, and tibia/fibula subset, depending on the inclusion of covariates (Table 2).
Survival analysis also found that increasing the average daily dose of PEMF treatment from 1 hour to 10 hours correlated with a reduction in the median time required to heal by $45 \%-50 \%$ in the 9-month scaphoid, tibia, and tibia/ fibula nonunion patient population. Moreover, the estimated reduction in the median time to heal for the 9-month tibia and tibia/fibula subset was $60 \%(P<0.0001)$.

Although PEMF dosage was the most important predictive variable for the time to heal, certain patient demographics/covariables were also found to have a minor correlation with the fracture healing time. Fracture site (scaphoid, tibia, and tibia/fibula), patient weight, and trophic level (oligotrophic, hypertrophic, and atrophic) were somewhat associated with the time to heal. Atrophic injuries tended to heal faster than oligotrophic or hypertrophic fractures, and scaphoid nonunions tended to heal faster than tibial nonunions. Conversely, patient age, sex, and the presence of motion at the fracture site did not correlate significantly with the time to heal.

\section{Discussion}

This study was the first to investigate whether there is a statistically significant correlation of average daily PEMF stimulation with the time required to obtain successful repair of nonunion fractures. Several different statistical evaluations concluded that increased average daily dose of PEMF stimulation correlates with a substantial and significant decrease in the required time to heal.

In terms of clinical efficacy, this study found that of the 1,382 patients evaluated, only 144 failed to heal, resulting in an overall clinical success rate of $89.6 \%$. For the audited patient population, the clinical success rate was $86.4 \%$. Several different clinical studies report similar clinical success rates for the Biomet ${ }^{\circledR}$ EBI Bone Healing System in the treatment of fracture nonunions. ${ }^{4-14}$ In 1990, a double-blind, randomized, placebo-controlled clinical trial was performed

Table 2 Survival analysis

\begin{tabular}{|c|c|c|c|c|}
\hline Fracture site & Disability & Median heal time reduction & Fractures (n) & $P$-value \\
\hline Scaphoid, tibia, and tibia/fibula & All nonunion fractures & $35 \%-40 \%$ & $369^{a}$ & $<0.000$ I \\
\hline Scaphoid, tibia, and tibia/fibula & 9-month nonunions & $45 \%-50 \%$ & 100 & $<0.0001$ \\
\hline Tibia and tibia/fibula & All nonunion fractures & $35 \%-40 \%$ & $317^{a}$ & $<0.0001$ \\
\hline Tibia and tibia/fibula & 9-month nonunions & $60 \%$ & 82 & $<0.0001$ \\
\hline Scaphoid & All nonunion fractures & $35 \%-40 \%$ & 52 & $0.001-0.015^{*}$ \\
\hline Scaphoid & 9-month nonunions & $17 \%$ & 18 & $0.3-0.7^{*}$ \\
\hline
\end{tabular}

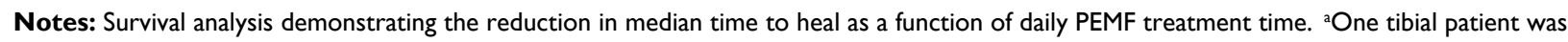
excluded from the survival analysis as the fracture site was not identified to be a tibia at the time this analysis was performed. *P-values shown depend on the number of covariates.

Abbreviation: PEMF, pulsed electromagnetic field. 
to evaluate the use of PEMF stimulation in the treatment of tibial fractures that had not healed after 16-32 weeks. ${ }^{13}$ Patients were treated with PEMF stimulation for 12 weeks and evaluated for their progress toward union. The results indicated that significantly more patients treated with PEMF stimulation progressed toward union than patients in the sham-treated control group. A 2-year follow-up of this study confirmed that $85 \%$ of the PEMF-treated fractures went on to heal without surgical intervention, as compared with $36 \%$ of the control patients. ${ }^{15}$ In addition, eight of the control patients opted for PEMF treatment following the end of the study, and six of the eight went on to heal without surgical treatment.

Preclinical research using the same PEMF therapeutic signal has also been conducted to better understand the effect PEMF stimulation has on the different cellular mechanisms involved with bone healing. The studies by Aaron and Ciombor ${ }^{16}$ and Aaron et $\mathrm{al}^{17}$ found that PEMF stimulation increases chondrogenesis and the production of transforming growth factor $\beta 1$ (TGF- $\beta 1$ ) leading to an increase in trabecular bone formation in a rat subcutaneous ossicle formation model. Rat calvarial osteoblasts were shown to have increased BMP-2 and BMP-4 expression with PEMF stimulation. ${ }^{18}$ Similarly, nonunion cells treated with PEMF stimulation were shown to increase the expression of TGF- $\beta 1 .{ }^{19}$ Furthermore, PEMF stimulation was shown to induce angiogenesis in human umbilical vein endothelial cells through an increased production in fibroblast growth factor $2 .{ }^{20}$ An evaluation of the effect of PEMF stimulation on the repair of mid-tibia osteotomies in canines revealed that PEMF stimulation resulted in a significant improvement in new bone formation and the resultant mechanical properties at the fracture site when compared with animals treated with sham controls. ${ }^{21}$ Overall, these different preclinical studies have shown that PEMF stimulation plays a role in several different cellular processes important for bone formation and ultimately leads to improved callus formation and maturation in late-phase bone healing.

Although earlier clinical and preclinical studies support the efficacy of PEMF stimulation in promoting bone healing, this study indicates, for the first time, that the time required to heal can be significantly affected by device usage and/or patient compliance. Since many factors can affect healing outcomes, alternative explanations for the dose-response relationship were also considered for this study. It is possible that the "dose-response" relationship itself may result from some other variables associated with the "dose". For example, the "dose" implies patient compliance with instruction, and it is probable that patients compliant with one aspect of their prescribed treatment regime (average daily PEMF stimulation), would also be compliant with other aspects of their prescribed treatment (eg, weight bearing). Although the absence of a randomized control group in this study limits the ability to separate the dose-response effects of PEMF stimulation from overall patient compliance, the ability of PEMF stimulation to induce fracture healing has been demonstrated in an earlier randomized, double-blind study that was not subject to compliance issues. ${ }^{13}$

\section{Conclusion}

This study shows a statistically significant correlation between average daily use of the Biomet ${ }^{\circledR}$ EBI Bone Healing System and a reduction in the average time to heal for nonunion fractures. A dose-response regression relationship was observed in which each additional hour of daily PEMF treatment was associated with a 6-day reduction in average time to heal. Survival analysis indicated that there was a $35 \%-60 \%$ reduction in the median time to heal for patients who used their device for an average of 10 hours per day when compared with patients who used their device for an average of 1 hour per day. In addition, patients who used PEMF stimulation for 9 hours or more per day healed on average 76 days earlier than patients who used PEMF stimulation for 3 hours or less per day. These different methods of statistical analysis all indicate that PEMF therapy is directly effective in inducing nonunion fracture repair and that increasing "dose" correlates with an acceleration in the rate of healing.

\section{Acknowledgment}

This study was conducted and funded by EBI, LLC (a Zimmer Biomet company).

\section{Disclosure}

The authors of this paper are current and former employees of EBI, LLC (a Zimmer Biomet company) and report no other conflicts of interest in this work.

\section{References}

1. United States Bone and Joint Decade. The Burden of Musculoskeletal Diseases in the United States: Prevalence, Societal and Economic Cost. Rosemont, IL: American Academy of Orthopaedic Surgeons; 2008.

2. Preamer A, Furner S, Rice D. Musculoskeletal Conditions in the United States. Park Ridge, IL: American Academy of Orthopaedic Surgeons; 1999.

3. Bio Osteogen System 204. EBI, LLC. US Food and Drug Administration premarket approval number: P790002. Decision date November 6, 1979 Available from: http://www.accessdata.fda.gov/scripts/cdrh/cfdocs/ cfpma/pma.cfm?id=P790002. Accessed August 19, 2016.

4. Adams BD, Frykman GK, Taleisnik J. Treatment of scaphoid nonunion with casting and pulsed electromagnetic fields: a study continuation. $J$ Hand Surg Am. 1992;17(5):910-914. 
5. Bassett CA, Mitchell SN, Gaston SR. Treatment of ununited tibial diaphyseal fractures with pulsing electromagnetic fields. J Bone Joint Surg Am. 1981;63(4):511-523.

6. Bassett CA, Mitchell SN, Gaston SR. Pulsing electromagnetic field treatment in ununited fractures and failed arthrodeses. JAMA. 1982; 247(5):623-628.

7. Bassett CA, Mitchell SN, Schink MM. Treatment of therapeutically resistant non-unions with bone grafts and pulsing electromagnetic fields. J Bone Joint Surg Am. 1982;64(8):1214-1220.

8. Dunn AW, Rush GA 3rd. Electrical stimulation in treatment of delayed union and nonunion of fractures and osteotomies. South Med J. 1984; 77(12):1530-1534.

9. Frykman GK, Taleisnik J, Peters G, et al. Treatment of nonunited scaphoid fractures by pulsed electromagnetic field and cast. J Hand Surg Am. 1986;11(3):344-349.

10. Holmes GB Jr. Treatment of delayed unions and nonunions of the proximal fifth metatarsal with pulsed electromagnetic fields. Foot Ankle Int. 1994;15(10):552-556.

11. Marcer M, Musatti G, Bassett CA. Results of pulsed electromagnetic fields (PEMFs) in ununited fractures after external skeletal fixation. Clin Orthop Relat Res. 1984;190:260-265.

12. Meskens MW, Stuyck JA, Feys H, Mulier JC. Treatment of nonunion using pulsed electromagnetic fields: a retrospective follow-up study. Acta Orthop Belg. 1990;56(2):483-488.

13. Sharrard WJ. A double-blind trial of pulsed electromagnetic fields for delayed union of tibial fractures. J Bone Joint Surg Br. 1990; 72(3):347-355.
14. Sharrard WJ, Sutcliffe ML, Robson MJ, Maceachern AG. The treatment of fibrous non-union of fractures by pulsing electromagnetic stimulation. J Bone Joint Surg Br. 1982;64(2):189-193.

15. Sharrard WJ. A double-blind trial of PEMF for delayed tibial union: clinical follow-up. Paper presented at: 16th Annual Meeting of the Bioelectromagnetics Society. Copenhagen, Denmark: 1994.

16. Aaron RK, Ciombor DM. Acceleration of experimental endochondral ossification by biophysical stimulation of the progenitor cell pool. J Orthop Res. 1996;14(4):582-589.

17. Aaron RK, Wang S, Ciombor DM. Upregulation of basal TGFbeta1 levels by EMF coincident with chondrogenesis - implications for skeletal repair and tissue engineering. J Orthop Res. 2002;20(2): 233-240.

18. Bodamyali T, Bhatt B, Hughes FJ, et al. Pulsed electromagnetic fields simultaneously induce osteogenesis and upregulate transcription of bone morphogenetic proteins 2 and 4 in rat osteoblasts in vitro. Biochem Biophys Res Commun. 1998;250(2):458-461.

19. Guerkov HH, Lohmann CH, Liu Y, et al. Pulsed electromagnetic fields increase growth factor release by nonunion cells. Clin Orthop Relat Res. 2001;384:265-279.

20. Tepper OM, Callaghan MJ, Chang EI, et al. Electromagnetic fields increase in vitro and in vivo angiogenesis through endothelial release of FGF-2. FASEB J. 2004;18(11):1231-1233.

21. Inoue N, Ohnishi I, Chen D, Deitz LW, Schwardt JD, Chao EY. Effect of pulsed electromagnetic fields (PEMF) on late-phase osteotomy gap healing in a canine tibial model. J Orthop Res. 2002;20(5): 1106-1114.
Orthopedic Research and Reviews

\section{Publish your work in this journal}

Orthopedic Research and Reviews is an international, peer-reviewed, open access journal that focusing on the patho-physiology of the musculoskeletal system, trauma, surgery and other corrective interventions to restore mobility and function. Advances in new technologies, materials, techniques and pharmacological agents are particularly

\section{Dovepress}

welcome. The manuscript management system is completely online and includes a very quick and fair peer-review system, which is all easy to use. Visit http://www.dovepress.com/testimonials.php to read real quotes from published authors. 